\section{Aggressivere Tumorstadien durch BPH-Arzneien?}

Trotz des Ergebnisses zweier großer Studien, dass die Behandlung mit einem 5-alpha-Reduktasehemmer das Gesamtrisiko für ein Prostatakarzinom um etwa $25 \%$ reduziert, gibt es aus den Studien auch Hinweise, dass das Risiko für die Entwicklung aggressiver Tumoren durch die Therapie erhöht wird. Koreanische Urologen sind dem nachgegangen.

W egen der Beobachtung in den Präventionsstudien PCPT (Prostate Cancer Prevention Trial) und REDUCE (Reduction by Dutasteride of Prostate Cancer Events), dass die Inzidenz von Prostatakarzinomen mit einem GleasonScore zwischen 8 und 10 absolut um $0,7 \%$ beziehungsweise $0,5 \%$ zugenommen hatte, hat die US-Zulassungsbehörde FDA im vergangenen Jahr die Beipackzettel zu Finasterid und Dutasterid durch den Hinweis auf dieses erhöhte Risiko ergänzen lassen. In einer kleinen retrospektiven Studie überprüften nun koreanische Urologen die Assoziation zwischen der Therapie mit 5-alpha-Reduktasehemmern und dem Gleason-Score bei Männern nach einer radikalen Prostatektomie.
Die Daten von insgesamt 1.204 Männern stammten aus dem Zeitraum zwischen 2003 und 2010. Nur 50 Männer gaben an, zur Therapie wegen einem benignem Prostatasyndrom (BPS) mindestens ein halbes Jahr lang mit einem 5-alpha-Reduktasehemmer behandelt worden zu sein: 36 Männer erhielten Finasterid, 14 Männer Dutasterid. Im Median dauerte die Therapie 23 Monate (mindestens sechs und höchstens 47 Monate). Im Median waren die Männer 66 Jahre alt (42 bis 82 Jahre) und hatten einen medianen PSA-Wert von $7,8 \mathrm{ng} / \mathrm{ml}$ (1,0 bis $84,4 \mathrm{ng} / \mathrm{ml})$. Bei $37 \%$ aller Studienteilnehmer lag das Prostatavolumen über $40 \mathrm{ml}$.

Nach Berücksichtigung mehrerer Faktoren wie Alter, BMI, PSA-Wert, Prosta- tavolumen und präoperativ ermitteltem Gleason-Score, errechneten die Ärzte mit einer Odds Ratio (OR) von 7,12 eine signifikante Assoziation zwischen der Therapie mit den 5-alpha-Reduktasehemmern und Gleason-Scores über $7 \mathrm{im}$ Vergleich zu den unbehandelten Männern. Zudem seien Tumorgewebe außerhalb der Prostata (OR: 2,5) sowie eine Infiltration der Samenblase (OR: 3,26) signifikant häufiger in den beiden Therapiegruppen aufgetreten.

Fazit: Die Beobachtung aus zwei großen placebokontrollierten Studien, dass die Therapie mit 5-alpha-Reduktasehemmern mit aggressiven Prostatatumoren assoziiert ist, wurde nun unabhängig auch in einer kleinen retrospektiven koreanischen Studie bestätigt. Für klare Aussagen zur Assoziation sind allerdings die Ergebnisse aus noch ausstehenden prospektiven Studien erforderlich.

Peter Leiner

Hong SK et al. Association of 5a-Reductase Inhibitor Use and Pathological Features of Prostate Cancer in Men Undergoing Radical Prostatectomy. Prostate 2012; 72: 1187-92

\section{PSA-Screening: $\operatorname{Man}(n)$ lebt nicht so viel besser wie länger}

In den Debatten ums PSA-Screening ging es bisher, überspitzt gesagt, um Leben oder Tod. Gesundheitsforscher haben die Frage nun anders formuliert. Sie wollten wissen: Macht der Test das Leben nicht nur länger, sondern auch besser?

D er European Randomized Study of Screening for Prostate Cancer (ERSPC) zufolge sinkt die ProstatakrebsSterblichkeit durch das PSA-Screening über elf Jahre betrachtet um knapp 30\%. Aus diesen Daten destillierten nun niederländische Forscher die Zahl der resultierenden qualitätskorrigierten Lebensjahre (QALY).

Pro 1.000 Männer zwischen 55 und 69 Jahren, die sich alle vier Jahre mit einer Teilnahmequote von $80 \%$ am PSA-Screening beteiligen, würde die Zahl der an Prostatakrebs Versterbenden um neun gesenkt (entsprechend einem Rückgang von $28 \%$ ). 14 Männern bliebe eine Palliativbehandlung erspart (-35\%). Insge- samt würden in einer Gruppe von 1.000 Männern 73 Lebensjahre hinzugewonnen - gut acht Jahre pro vermiedenem Prostatakrebstod. Doch die Zahl der gewonnen QALYs läge nur bei 56, das sind $23 \%$ weniger als die zusätzlichen Jahre insgesamt. Dies liegt daran, dass die postdiagnostischen Folgen der Therapie und vor allem auch der Übertherapie mit in die Rechnung einfließen. Denn je nachdem, ob die Jahre in Potenz oder Impotenz, Inkontinenz oder Kontinenz verlebt werden, ist ihre Qualität höher oder niedriger zu veranschlagen. Statistisch ergibt sich eine breite Streuung. So kann es sein, dass dem Screening statt der 56 ganze 97 QALYs zu danken sind. Es kann jedoch auch dazu kommen, dass 21 QUALYs verloren gehen.

Die Bewertung des Nutzens, den Männer einzelnen Lebensphasen beimessen würden, ist allerdings diffizil. Erektile Dysfunktion nach Prostatektomie mag den einen grämen, während sich ein anderer mit den übrigen Freuden des gewonnen Lebens tröstet. In jedem Fall aber zeigen die Ergebnisse, dass vor einer generellen Screeningempfehlung die Folgen für die Lebensqualität noch besser erforscht werden müssen.

Fazit: Folgt man den Modellrechnungen des Erasmus Medical Center in Rotterdam, wird das Leben durch die Teilnahme am PSA-Screening wahrscheinlich nicht so viel besser wie es länger wird. Und es ist nicht einmal ausgeschlossen, dass es sich verschlechtert. Dr. Robert Bublak

Heijnsdijk EAM et al. Quality-of-Life Effects of Prostate-Specific Antigen Screening.

N Engl J Med 2012; 367: 595-605 